\title{
Drei Wochen Erfahrungen in Pakistan
}

Gebet auf Trümmern: In Balakot (50 000 Einwohner) hielt dem Erdbeben nur ein einziges Gebäude stand.

Foto: Keystone

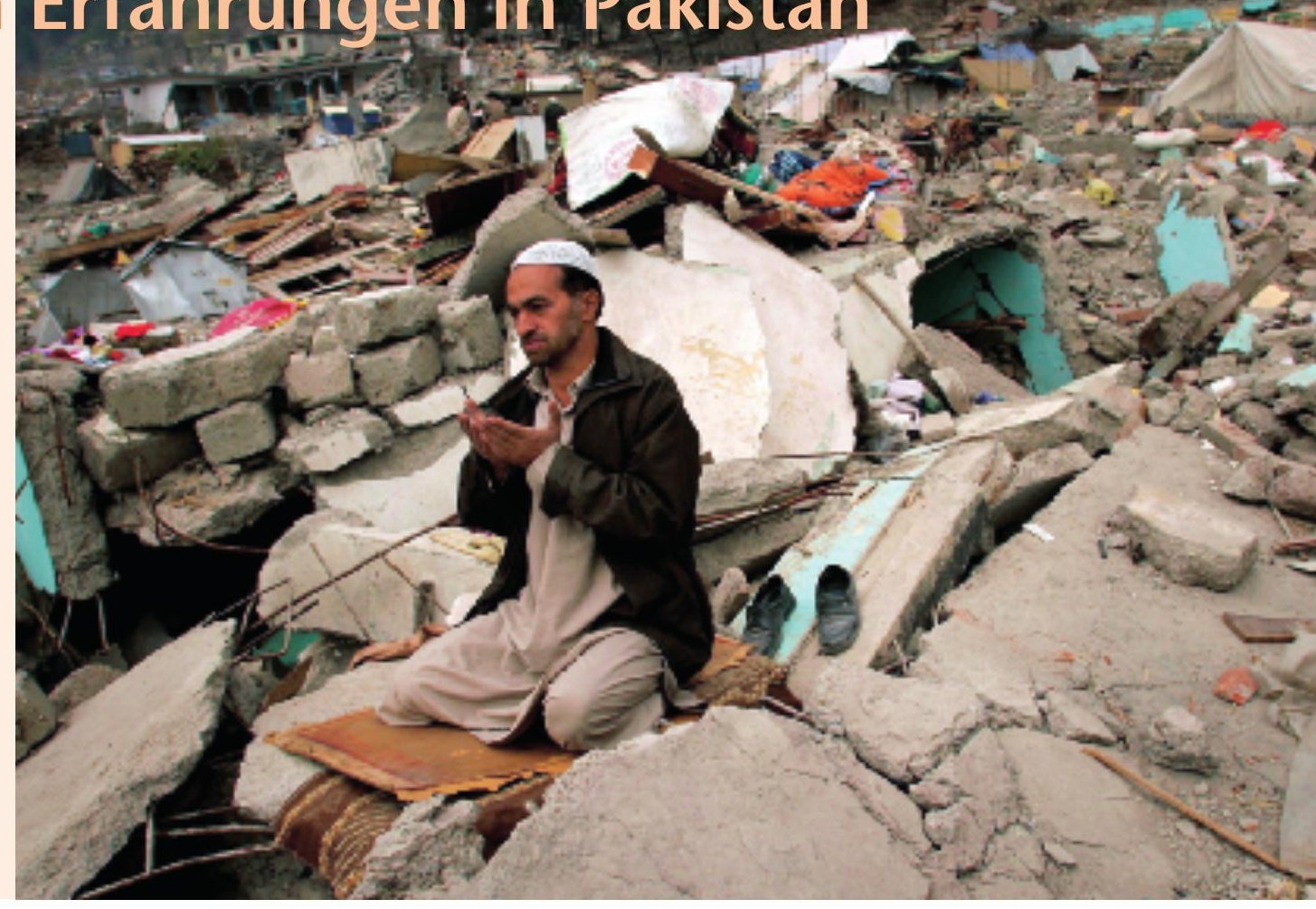

Gisela Perren-Klingler

Korrespondenz:

Dr. med. Gisela Perren-Klingler Napoleonstrasse 16b

CH-3930 Visp

iptsperren@rhone.ch
Am 8. Oktober 2005 ist Pakistan von einem schweren Erdbeben heimgesucht worden. Im Sommer vorher hatte ich an einem Psychotraumatologiekongress mit einer pakistanischen Kollegin, mit der ich seit mehr als 20 Jahren befreundet bin, gesprochen, so z.B. unter anderem über frühe Interventionen nach Exposition (wir sprachen hauptsächlich von familiärer Gewalt), therapeutische Ansätze später und nach Ausbrechen einer PTBS. Kurz nach dem Erdbeben schrieb sie mir, dass sie meine Hilfe brauche; zwar bestehe viel Hilfsbereitschaft unter Kollegen, dass sie aber nicht genau wüssten, was sie tun könnten/sollten. Da es im Rahmen meines Pensums keine andere Möglichkeit gab, entschieden wir uns - auch in der Familie-, dass ich über die Weihnachtstage für etwa drei Wochen in das betroffene Gebiet nach Pakistan flöge. Als ehemalige Psychiaterin der Pakistan International Airline, PIA, meldete mir die Kollegin, dass man auch gerade eine Ausbildung für CareTeam-Mitglieder («Family Assistance Teams») plane, und ob ich dies auch übernehmen könne. Ich nahm diesen Vorschlag an, da ich vor einiger Zeit bei der Ausbildung solcher Teams in der Lufthansa mitgewirkt habe. Mein Programm in Pakistan bestand nun aus zwei Teilen: Einerseits sollte ich dreimal während zweier Tage je 30 Freiwillige der PIA ausbilden, und dann sollte ich zweimal während dreier Tage Kollegen und Freiwillige im Umgang mit Erdbebengeschädigten ausbilden.

Die Arbeit bei PIA ermöglichte mir, kurzfristig zu einem Flugticket zu kommen und so trotz ausgebuchten Fliegern «gratis» nach Pakistan und zurückzufliegen. Diese Aktivitäten sind hier aber nicht relevant.

Nach meiner Rückkehr möchte ich über die beiden Kurse mit Helfern für die Betroffenen, über meine eigenen «Felderfahrungen» und dabei aufgetretene Fragen berichten.

\section{Karachi}

Der erste dreitägige Kurs fand in Karachi statt, in den Räumen der PMA (Pakistan Medical Association). Pausen- sowie Mittagsverpflegung wurde durch den Vertreter der Firma Hoffmann-La Roche geleistet, die Handouts wurden von der Firma Eli Lilly vervielfältigt.

Im ersten Kurs nahmen verschiedene Psychiater der Stadt teil, darunter ein Associate Professor der Agha Khan University, Psychologen/-innen, jüngere Kollegen, die in der Ausbildung zum Psychiater sind, eine Pädiaterin, die im Erdbebengebiet arbeitet, und drei Lehrerinnen, insgesamt 24 Personen.

Ziel der Arbeit war, den Teilnehmern theoretisches Grundwissen über eine salutogenetische 
Sicht von Trauma zu übermitteln, Unterschiede zwischen normalen und pathologischen Reaktionen wahrzunehmen und eine gewisse kritische Rezeption des Traumabegriffes (Angsterkrankung versus dissoziative Erkrankung) zu erarbeiten.

Auf der praktischen Ebene sollten die Teilnehmer befähigt werden, kognitiv eine Narrative des jeweils persönlich Erlebten mit den dazugehörigen Gefühlen und eine salutogenetische Erklärung (im Sinne von Reframings) dafür zu erarbeiten. Ebenfalls wurde geübt, wie man Stressmanagementtechniken selber anwendet, diese den Klienten/Patienten beibringt und sie dann dazu motiviert, sie täglich selbständig und konsequent durchzuführen. Im Hintergrund stand die Überlegung, dass diese Kolleginnen und Kollegen alle freiwillig einmal pro zwei Monate eine Woche im Gebiet des Erdbebens verbringen würden, um so ihre religiöse Verpflichtung, Almosen (Zakaat) zu geben, einzulösen. Damit war klar, dass man nicht im Rahmen einer «Therapie» von traumatischen Reaktionen, Trauer oder PTBS denken konnte, sondern sich überlegen musste, wie man den Betroffenen soviel wie möglich an Bewältigungskompetenz und -strategien übermitteln könnte. Ebenso war es wichtig, neben dem allgemeinen lokalen Glauben an die Effizienz von Psychopharmaka bei traumatischen Reaktionen und bei PTBS noch andere Möglichkeiten aufzuzeigen, die mehr auf der Ebene der Sprache und dem Fördern von Bewältigungsfähigkeiten beruhen.

Viel Neugier wurde gerade auch von den Kollegen eingebracht, und sie konnte teilweise befriedigt werden, indem ich auch über die «neuesten» Techniken wie EMDR, TFT, EFT usw. berichtete - allerdings in einem transkulturellen Rahmen: Es gibt ja seit sehr langer Zeit in andern Kulturen verschiedene Techniken der bilateralen Stimulation nach «Schreckerfahrungen», so z. B. in der muslimisch-bosnischen Tradition und in Zentralamerika.

Es wurde ebenfalls die von Basoglu entwickelte Technik der einmaligen CBT-orientierten Intervention, die er bei Patienten mit PTBS nach dem Erdbeben in der Türkei angewandt und überprüft hat, eingebracht. Seine längerfristig angelegte statistische Evaluation zeigt eine deutliche Abnahme der Symptomatik von PTBS, so dass nach zwölf Monaten $85 \%$ der Behandelten über sehr stark und stark verbesserte PTBSbedingte Einschränkungen berichten. Basoglu erwähnt eigens, dass dieses Modell für Orte geeignet sein könnte, in denen die Psychiaterdichte so minimal ist, dass man an keine längere Behandlung denken könne. Seine Behandlungs- anweisungen sind so konzipiert, dass es für Studenten der Psychologie möglich ist, nach einer kurzen Einführungszeit diese einmalige Intervention effizient zu tätigen.

Basoglu legt viel Wert darauf, dass die Vermeidungsreaktionen bekämpft werden müssen. Die von ihm gewählte Metapher besteht darin, dass man gegen die Angst wie gegen einen Feind kämpft mit der Absicht, ihn zu besiegen: Wer durch Exposition täglich gegen die Angst kämpft, wird auf Dauer gewinnen. Die gezielte Aufhebung des Vermeidungsverhaltens, das als bester prädiktiver Faktor für die Entwicklung von PTBS gilt, scheint besonders nach Erdbeben wichtig zu sein. Dieses Modell Basoglus steht dem von mir gelehrten Modell am nächsten: In Europa benütze ich die Metapher des «Scheinriesen», die den meisten Leuten aus der Kindergeschichte Michael Endes von «Jim Knopf und Lukas der Lokomotivführer» bekannt ist.

Die Anpassung der verschiedenen Techniken bei der Arbeit mit Kindern einerseits und ihre Einbettung in die jeweiligen Systeme (Schule, Familien, Heime) andererseits wurden ebenfalls besprochen.

Speziell legte ich von der ersten Stunde an viel Wert auf das Training von Selbstschutz - mit theoretischen und praktischen Überlegungen sowie Tips und Tricks, damit die Teilnehmer sich bei der Arbeit mit Betroffenen effizient schützen lernten.

Nach drei Tagen intensiver und disziplinierter Arbeit schienen alle Teilnehmer zufrieden: Sie hatten Altbekanntes und Neues angetroffen und waren überzeugt, dass sie sich bei ihrer weiteren Arbeit im Erdbebengebiet besser schützen und dem Wort etwas mehr Platz einräumen wollten.

\section{Abbottabad}

Der zweite Kurs fand im Erdbebengebiet selber statt, und zwar im Women's Medical College in Abbottabad, einer auf etwa $1200 \mathrm{~m}$ gelegenen kleinen Distrikthauptstadt. An diesem Kurs nahmen keine Psychiater teil, einige wenige Psychologinnen, dafür drei Vertreter der «Community Medicine», mehrere Sozialarbeiter und Lehrer und freiwillige Helfer des lokalen Lyons Klubs, im ganzen 27 Personen. Damit musste der Inhalt des Kurses total verändert und die Theorie von Trauma und seinen möglichen psychologischen Folgen vereinfacht und dieser Zielgruppe angepasst werden.

Die Arbeit war für mich schwieriger, da viele der Teilnehmer nur über rudimentäre Englischkenntnisse verfügten, weshalb immer wieder übersetzt werden musste, und zwar von Ad-hocÜbersetzern, was die Genauigkeit der Über- 
setzung nicht immer garantierte. Der Inhalt blieb im Prinzip der gleiche wie in Karachi.

Die Erfahrung der Gruppe wurde in einer Schlussrunde einzeln wiedergegeben - allerdings meist in Urdu, so dass ich wieder auf eine Übersetzung angewiesen war ...

Als Beispiel zitiere ich die Aussage eines Schuldirektors, die seine Lernerfahrung wiedergibt: Seine Schüler aus den erdbebenverwüsteten Dörfern seien nach dem 8. Oktober so seltsam gewesen und ihr Verhalten unverständlich. Sie hätten die Schulstunden mit dem gepackten und auf dem Rücken bleibenden Schulranzen verbracht, mit permanent hoher Aufmerksamkeit auf eventuelle Nachbeben und immer auf dem Sprung, sobald es wieder etwas rüttelte. Nun sei ihm die Bedeutung ihres Verhaltens klar geworden. Er werde diese Einsicht seinen Lehrern beibringen, ebenso, wie man Stressmanagement in der Schule tätigen könne, um trotz der vielen Nachbeben ruhiger (und vielleicht angstfreier) weiterarbeiten zu können.

\section{«Felderfahrung»}

Natürlich konnte ich nicht nur im Womens Medical College ein- und ausgehen, sondern ich musste auch den von mir so gefürchteten «humanitären Tourismus» an verschiedene Orte mitmachen.

Was auf einer Fahrt nach Balakot, einer dem Erdboden gleichgemachten Stadt an einem kleinen Fluss, auf dem Weg nach Gilgit - 700 km von der Grenze zu China entfernt, am meisten ins Auge stach, waren einerseits die frischen Gräber, die immer wieder an der Strasse zu sehen waren, und andererseits die terrassierten und offensichtlich eingesäten Felder bis in Balakot, wo neben den Trümmern von Häusern bereits bestellte terrassierte kleine Felder zu sehen waren.

In Balakot (50000 Einwohner) ist von der ganzen Stadt nur ein einziges Gebäude stehengeblieben. Der Rest der Stadt besteht noch aus kleinen Betonresten, aus denen spärliche Eisenarmierungen hervorschauen. Diese werden, wie alles andere wiederverwertbare Material, herausgelöst und auf improvisierten Waagen gewogen und dann verkauft. Grosse Caterpillarmaschinen sind bereits dran, die Überreste der Häuser zusammenzuschieben und den Boden für Neukonstruktionen freizumachen. Es bleibt zu hoffen, dass die neuen Häuser erdbebenkonform mit mehr Eisenarmierungen erbaut werden und so die einzige funktionierende Primärprävention gegen Erdbeben geleistet wird.

In Balakot sind in zwei Schulen mehr als 500 Kinder und Jugendliche umgekommen, da das Erdbeben gegen 8.30 Uhr stattfand, als die
Schule bereits begonnen hatte. Es gibt Familien, die alle oder fast alle Kinder verloren haben, daneben gibt es auch Kinder, die ihre Eltern verloren haben. Diese Waisenkinder wurden sofort von verschiedenen Organisationen, die sich als Adoptions-NGOs vorgaben, zur Adoption vorbereitet. Die Regierung reagierte umgehend, so dass bis auf weiteres keine Vollwaisen aus dem Erdbebengebiet adoptiert werden können. Sie sind in SOS-Kinderdörfern untergebracht.

Neben dem geschäftigen Treiben - es gab auch schon wieder einen Stand, an dem man auf einem Kocher gemachten Tee kaufen konnte mutete einen, fast unheimlich, das Schweigen oder der niedrige Lärmpegel an, ganz ungewöhnlich für Pakistan. Eine Hilfskrankenschwester schilderte mir das gleiche bei ausgeflogenen verletzten Patienten, bei denen sie durch das unheimliche Schweigen und die Wortlosigkeit der frisch Operierten in bezug auf Schmerzen, Bedürfnisse (wie Essen, aufs Klo gehen usw.) frappiert war, und bei denen das Personal lernen musste, präzise nachzufragen, um den Patienten gerecht zu werden.

Ein anderer Ausflug führte in eine Zeltstadt neben Abbottabad, auf das Bauland für die Erweiterung der Universität: Die ersten 150 Wellblechcontainer waren sofort nach dem Erdbeben vom lokalen Lyons Klub aufgestellt worden. Einer meiner Kursteilnehmer, Jurist und Dozent für Recht an der Islamic University in Abbottabad, berichtete mir, dass er täglich die ungefähr $15 \mathrm{~km}$ dorthin fahre, um zu sehen, ob alles funktioniert. Neben «seinen» 150 Containern - pro Grossfamilie einer - hat nun das UNHCR (Hochkommissariat für Flüchtlinge) weitere 1000 Zelte aufgestellt. Während der Lyons Klub für seine Container islamkonforme Nasslatrinen mit Abfluss ins nahegelegene Wadi errichtet hat, hat OXFAM (eine grosse englische NGO) für die UNHCR-Zeltstadt Trockenlatrinen ohne Abfluss aufgestellt, die nun bereits bald voll und sehr unbeliebt sind, was bedeutet, dass die Leute ihre Bedürfnisse in der Natur befriedigen ...).

Neben den Wohnzelten gibt es Schulzelte, Kindergartenzelte, ein Zelt für die Basisgesundheitsversorgung und das Verwaltungszelt. Der Verwalter der Zeltstadt konnte mir anhand seines handgeschriebenen Heftes über die untergebrachten Familien genau sagen, wie viele Kinder (3-16 Jahre) und wie viele Erwachsene im Lager sind, nach Geschlecht geordnet, und wie viele Säuglinge (0-3 Jahre) es gibt - insgesamt etwas über 6000 Menschen. Der Verwalter ist vom Ministerium für Soziales aus Abbottabad in diese Zeltstadt abgeordnet worden. Die Sicherheit wird von Soldaten gewährleistet. Auf meine 
Frage hin sagte der Verwalter, dass es in der Zeltstadt weder Diebstahl noch Vergewaltigung gäbe. Das schwierigste sei im Moment, für so viele Menschen Nahrung zu bereiten, und als nächstes würden nun Kocheinrichtungen kommen, damit die Frauen dann in einer Art Grossküche gruppenweise sich selber mit dem Kochen befassen könnten/müssten. Das Einkaufen solle dann durch die Männer übernommen werden d.h. die Selbständigkeit der Menschen solle schon jetzt gefördert werden. Von Regierungsseite ist bereits geplant und wird permanent mitgeteilt, dass ab April die Zeltstädte geschlossen werden sollen. Sie sollen der Bergbevölkerung nur helfen, während der Zeit des Schnees im milderen Klima von Abbottabad zu überwintern.

Die Regierung hat einen schweren Stand bei der Bergbevölkerung: Kurz nach dem Erdbeben ging das Gerücht um, dass die Regierung vom bevorstehenden Erdbeben gewusst habe, das Wissen aber für sich behalten habe und damit für die vielen Toten verantwortlich sei.

Deswegen gab es viele Fernseh- und Radiosendungen in den regierungseigenen elektronischen Medien, in denen die Bevölkerung über Ursachen von Erdbeben, aber auch über den Umgang mit psychischen Folgen von Schock, Schreck und Trauer aufgeklärt wurde. Die Kollegin hatte da als ärztliche Lehrerin mitgewirkt.

Meine Kollegin wohnte mit ihren Mitarbeitern - alle bezahlten ihren Flug von Karachi nach Islamabad selber und leisten dort Freiwilligenarbeit - auf dem Militärcompound. Ich durfte als Ausländerin nicht dort wohnen und musste so in einem Hotel in Abbottabad wohnen.

Jeden Tag wurden ihr Patienten zur psychiatrischen Kontrolle gebracht: Ich bot ihr an, nach meinem Kurs dabei behilflich zu sein. Einer ihrer Mitarbeiter, ein junger Mediziner, der sich in Psychiatrie spezialisieren will, diente mir als Übersetzer.

An einem Abend sahen wir zusammen neun Patienten: Eine Frau hatte seit dem Erdbeben Angst, in ihrem Haus zu schlafen. Ihr wurde die Vermeidungsstrategie erklärt. Mittels einer einfachen Atemübung wurde ihr beigebracht, wie sie wieder zur Ruhe gelangen kann. Dadurch lernte sie, den durch die Konfrontation entstehenden Stress besser zu managen, so dass sie jeden Tag wieder ins Haus gehen und dann auch dort wieder schlafen kann - die Metapher Basoglus leuchtete ihr ein.

Diese Reaktion war mir von Basoglus Beschreibungen nach dem Erdbeben in der Türkei bekannt, und ich konnte sie auch in Pakistan von der Strasse aus immer wieder bestätigt sehen: Neben nichtzerstörten, d.h. gutgebauten Häu- sern gab es Zelte, da die Hausbewohner fürchteten, in ihren Häusern zu schlafen, obwohl sie ja dem Haupterdbeben widerstanden hatten, eine typische Vermeidungsreaktion.

Ansonsten hatten wir es ausschliesslich mit Patienten zu tun, die bereits von einem lokalen Psychiater behandelt - und leider immer mit Medikamenten vollgestopft - waren. In einer ethisch eigentlich kaum zu gewinnenden Situation versuchte ich, im Gespräch mit jedem Patienten seine Ressourcen zu aktivieren und ihn darauf hinzuweisen, dass er - neben der Medikation - Dinge für sich tun müsse - egal, wie schwierig dies sei. Es handelte sich um individuell angepasste und gezielte Verhaltensmassnahmen, die hauptsächlich bei Angstpatienten eine erfolgreiche Wirkung erzielen konnten. Ich enthielt mich also, die teilweise sehr hohe Dosierung von und die Kombination mit verschiedenen Psychopharmaka zu verändern, da ja keine Nachhaltigkeit geboten werden konnte. Die freiwilligen Organisatoren - aus dem Lyons Klub - waren ganz erstaunt über das Echo: Die Menschen seien alle zufrieden gewesen, obwohl ich keine andern Medikamente verschrieben hätte.

An einem andern Abend bekamen wir hauptsächlich Psychotiker aus einem Dorf zu sehen. Der Wahn eines Patienten drehte sich um seine Kontakte mit Bin Laden und seinen Wunsch, in den Jihad zu ziehen. Ich motivierte ihn, seine Medikamente regelmässig zu nehmen, da er sonst nicht als Kämpfer in Frage komme ... In diesem Dorf schockierte mich aber ein Bild in einem Haus, auf welchem zwei Jungen, 6 und 8 Jahre, in Tarnuniform und mit einem Gewehr im Arm gezeigt wurden. Der Titel darunter lautet: «Proud to be Islamic Fundamentalists».

\section{Reflexion}

Die für mich wichtigste Aufgabe, einen PublicMental-Health-Zugang zu planen, d.h. Lehrern und Sozialarbeitern sowie eventuell Pädiatern und Ärzten der Grundversorgung einige Anhaltspunkte für die Diagnostik akuter traumatischer Reaktionen und der PTBS zu vermitteln, den einfachen Zugang übers Narrative aufzuzeigen, Psychoedukation in bezug auf Vermeidung und Stressmanagement einzuüben, blieb m. E. in der Praxis auf der Strecke. Die Psychiaterin und ihr Team konnten sich nicht von ihrem individuumzentrierten und therapeutisch ausgerichteten Ansatz aus der Psychopathologie lösen. Das Paradigma der Salutogenese war zwar bekannt, aber es konnte nicht umgesetzt werden. $\mathrm{Zu}$ viele individual-therapeutische Reflexe stehen dem im Weg. Das geht soweit, dass eine Gruppe z. B. 
einmal während zweier Stunden in einem Lager «therapeutisch» mit Kindern bastelt - und auch ein paar Worte wechselt -, um vielleicht in zwei Monaten das gleiche zu wiederholen. Spätestens da musste man sich die Frage nach der ethischen Dimension der gängigen Interventionen stellen, «primum nihil nocere». Daraus ergibt sich für mich eine Reihe von Anfragen: Was bedeutet es für Kinder im Kindergartenalter oder in der frühen Primarschule, wenn einmal alle zwei Monate zwei «Städter» kommen und mit ihnen etwas basteln? Wäre da das Geld nicht sinnvoller ausgegeben, wenn man Fussbälle und anderes Material für Sport und Spiele ausgegeben hätte? Und werden diese Kollegen nun in den nächsten Monaten alle temporär dislozierten Dorfbewohner aus den Snowlands hauptsächlich mit Psychopharmaka beglücken? Wer fördert die Bewältigungsfähigkeit und Resilienz der Bevölkerung? Und wer ermöglicht es, dass die Bevölkerung nicht mit religiösen Gerüchten be- lastet wird, wie z.B., das Erdbeben habe wegen ihrer Sünden stattgefunden?

Auch wenn für mich die drei Wochen in Pakistan sehr interessant waren und viele Begegnungen mit sehr verschiedenen Menschen brachten, mir eine neue Dimension des auch von Akademikern gelebten pakistanischen Islams auftaten, haben sich viele Fragen aufgetan: Wieviel Zeit und Training braucht es, um meist in England ausgebildete - Psychiater dahin zu bringen, dass sie - nur temporär - von ihrer pathologiezentrierten und damit therapeutisch gedachten, auf das Individuum ausgerichteten und damit die Ressourcen der Gemeinschaft ignorierenden Haltung wegkommen? Meine anfängliche Annahme, die «Kollegin in Not» sei fähig, therapeutische Reflexe auf die Seite zu legen, um in der Not anders zu funktionieren, war falsch. Damit ist die Garantie dafür nicht gegeben, dass eine gewisse Nachhaltigkeit meiner Investition an Zeit und Energie entspricht. 